\title{
Prevalencia y experiencia de caries dental en estudiantes según facultades de una universidad particular peruana
}

\author{
Prevalence and experience of dental caries in different faculty students at peruvian private university
}

Miguel Espinoza Solano ${ }^{1 a}$, Roberto Antonio León-Manco ${ }^{1 a, 2 b}$

\section{RESUMEN}

Objetivos: Describir la prevalencia y experiencia de caries dental en estudiantes según facultades de la Universidad Peruana Cayetano Heredia (UPCH), Lima-Perú, en el año 2012. Material y métodos: Estudio descriptivo, observacional y retrospectivo, se trabajó con datos 2 bases de datos (Base 1: 3454 registros y Base 2: 3417 registros) provenientes de estudiantes de la UPCH proporcionados por el área administrativa de la Clínica Dental Docente de la misma institución. Las variables de estudio fueron la prevalencia y experiencia de caries dental (Índices CPOD y CPOS) según facultades,las pruebas estadísticas utilizadas fueron Chi-cuadrado y Kruskall Wallis, contando con un nivel de confianza de 95\% y p<0,05, se usó el programa estadístico SPSS v. 20.0. Resultados: Se encontró asociación de la prevalencia de caries dental $(\mathrm{p}<0,01)$ y diferencia de promedios de Índices CPOD y CPOS $(\mathrm{p}<0.01)$ según facultades en la UPCH. Conclusiones: Se evidenciaron diferencias estadísticamente significativas entrela prevalencia y experiencia de caries dental en estudiantes según facultades de la Universidad Peruana Cayetano Heredia, Lima-Perú, en el año 2012.

PALABRAS CLAVE: Epidemiología, caries dental, determinantes sociales de la salud, gradiente social.

\section{SUMMARY}

Objectives: Describe the prevalence and experience of dental caries in students on faculty at the Universidad PeruanaCayetano Heredia (UPCH), Lima, Peru, in 2012. Materials and Methods: A descriptive, observational and retrospective study, worked with records of 2 data bases (Base 1: Base 23454 records: 3417 records) students from UPCH provided by the administrative area of the Teaching Dental Clinic of the same institution. The 
variables were the prevalence and experience of dental caries (DMFT and DMFS indices) as faculties, statistical tests used were Chi-square and Kruskall Wallis, with a confidence level of $95 \%$ and $p<0.05$, the program was used SPSS statistical v. 20.0. Results: Association of the prevalence of dental caries $(p<0.01)$ and mean difference of DMFT and DMFS indices ( $p$ <.01) was found as faculties in UPCH. Conclusions:Statistically significant differences between the prevalence and experience of dental caries in students is evidenced by faculties of the Universidad PeruanaCayetano Heredia, Lima - Peru, in 2012.

\section{KEYWORDS: Epidemiology, dental caries, social determinants health, social gradient.}

\section{INTRODUCCIÓN}

Una de las enfermedades buco dentales más frecuentes es la caries dental, según la Organización Mundial de la Salud (OMS) afecta entre un 60\% a $90 \%$ de los niños en edad escolar entre 5 a 17 años, según el reporte de la Organización Panamericana de la Salud (OPS) para esta población, el Î́ndice CPOD varía entre 1,08 a 8,3 con un promedio de 4,4 , estos valores muestran problemas aún no resueltos (1-3).

En el Perú, la caries dental también es una de las enfermedades de mayor prevalencia, aumentando la gravedad según la edad de los individuos; por lo tanto, la necesidad de tratamiento tendrá un mayor costo al paso de los años siendo producto de una deficiente salud bucal en sus primeros años de vida producto de la limitada accesibilidad a los servicios de salud y las inadecuadas prácticas en prevención de enfermedades orales $(4,5)$.

El presente estudio tuvo como propósito medir la prevalencia y experiencia de caries dental en estudiantes según facultades de la Universidad Peruana Cayetano Heredia en el año 2012.

\section{MATERIAL Y MÉTODOS}

El presente estudio tuvo un diseño descriptivo, observacional y retrospectivo. La muestra y población fue la misma, siendo registros pertenecientes a dos bases de datos de la Clínica Dental Docente de la Facultad de Estomatología Roberto Beltrán de la UPCH, correspondientes al examen odontológico anual que se realizó a los estudiantes el año 2012, según registros se evaluaron un total de 3474 estudiantes y los resultados se organizaron en dos bases de datos, la primera con registros de prevalencias de caries dental, y la segunda con los datos de experiencia de caries dental (excluyéndose registros incom- pletos, se obtuvieron; Base 1: 3454 registros y Base 2: 3417 registros). Las variables de estudio fueron la prevalencia de caries dental, y experiencia de caries dental medida mediante los Índices CPOD (número de dientes permanentes cariados, perdidos y obturados) e Índice CPOS (número de superficies de dientes permanentes cariados, perdidos y obturados), se tuvo como co-variables las diversas facultades de la Universidad: Ciencias y Filosofía, Enfermería, Estomatología, Medicina, Medicina Veterinaria y Zootecnia, Psicología, Salud Pública y Administración, y Tecnología Médica, esta última es una escuela profesional dependiente de la Facultad de Medicina, pero para el estudio se ha considerado como una facultad diferenciada por ser una carrera distinta y el significante volumen de estudiantes.

Se solicitó permiso para acceder a los datos epidemiológicos de los estudiantes de la Universidad Peruana Cayetano Heredia del año 2012 mediante una carta dirigida al Jefe encargado del área de diagnóstico de la Clínica Dental Docente Cayetano Heredia. Con los registros de las bases de datos se procedió a la depuración de la misma, se digitaron los datos en el programa Microsoft Excel 2010 para su posterior análisis en el programa estadístico SPSS v 20.0. Para el análisis univariado se obtuvieron las frecuencias absolutas y relativas de la prevalencia de caries dental, y, los promedios y desviación estándar del Î́ndice CPOD y CPOS, en forma general y por facultades. Para el análisis bivariado de la prevalencia de caries dental se utilizó la prueba de Chi-cuadrado, y para los índices de CPOS y CPOS se empleó la prueba no paramétrica de Kruskall Wallis, debido a que los datos siguen una distribución z siendo comprobada mediante la prueba de Kolmogorov-Smirnov, el presente estudio contó con un nivel de confianza de 95\% y $\mathrm{p}<0,05$.

La investigación fue aprobada por el Comité de 
Institucional de Ética de la Universidad Peruana Cayetano Heredia (CIE-UPCH) el 19 de Mayo de 2014.

\section{RESULTADOS}

En general, la prevalencia de caries dental fue de 71,2\% (n=2458), observándose la prevalencia más alta en la Facultad de Enfermería (82,0\%; n=305) y la más baja en la Facultad de Estomatología (60,5\%; n= 319). Se evidenció una asociación entre la prevalencia de caries dental y las diversas facultades $(\mathrm{p}<0,01)$ (Tabla 1).
En la evaluación global de los estudiantes se obtuvo un promedio de Índice CPOD de 4,7 $(\mathrm{DE}=3,5)$ y un CPOS de 7,3 ( $\mathrm{DE}=6,2)$, en este caso, el mayor promedio de índice CPOD lo reportó la Facultad de Estomatología $(X=5,0 ; \mathrm{DE}=3,4)$ y el menor correspondió a la Facultad de Veterinaria y Zootecnia $(\mathrm{X}=4,1 ; \mathrm{DE}=3,0)$. En el caso del CPOS, el mayor promedio lo presentó la Facultad de Salud Pública y Administración $(X=11,1 ; \mathrm{DE}=11,7)$ y el menor valor lo tuvo la Facultad de Veterinaria y Zootecnia $(\mathrm{X}=6,4$; $\mathrm{DE}=5,5)$. Se encontró diferencia estadísticamente significativa del promedio de ambos índices según facultades $(\mathrm{p}<0,01)$ (Tabla 2).

Tabla 1. Prevalencia de caries dental en estudiantes de la Universidad Peruana Cayetano Heredia, Lima-Perú, en el año 2012.

\begin{tabular}{lrrrrrl}
\hline \multirow{1}{*}{ FACULTADES } & POBLACION & \multicolumn{2}{c}{ SANOS } & \multicolumn{2}{c}{ ENFERMOS } & \multirow{2}{*}{ p } \\
& N & n & \% & n & \% & P \\
\hline & & & & & & \\
Ciencias y Filosofía & 435 & 157 & 36,1 & 278 & 63,9 & \\
Enfermería & 372 & 67 & 18,0 & 305 & 82,0 & \\
Estomatología & 527 & 208 & 39,5 & 319 & 60,5 & \\
Medicina & 1119 & 328 & 29,3 & 791 & 70,7 & \\
Medicina Veterinaria y Zootecnia & 272 & 79 & 29,0 & 193 & 71,0 & $<0,01^{*}$ \\
Psicología & 164 & 52 & 31,7 & 112 & 68,3 & \\
Salud Pública y Administración & 64 & 14 & 21,9 & 50 & 78,1 & \\
Tecnología Médica & & & & & \\
Total & 501 & 91 & 18,2 & 410 & 81,8 & \\
\hline
\end{tabular}

N: Población total.

n: Frecuencia absoluta.

$\%$ : Frecuencia relativa.

○: Escuela profesional dependiente de la Facultad de Medicina.

p: Significancia estadística.

*: Prueba de Chi-cuadrado.

Base de Datos 1: 3454 registros de estudiantes.

\section{DISCUSIÓN}

La caries dental es una enfermedad multifactorial, crónica y prevenible, donde interactúan: la dieta, compuesta por los carbohidratos fermentables, la microflora, el huésped, así como factores externos (nivel socioeconómico) y también aquellos factores internos (la susceptibilidad del diente, flujo y capacidad tampón de la saliva, higiene bucal, factores hereditarios y estado nutricional entre otros). Esta enfermedad afecta a millones de personas desde la infancia, es por ello que la OMS define a la caries dental como el reblandecimiento del tejido duro del diente que va evolucionando hasta la formación de una cavidad, afectando la salud general y la calidad de vida de los individuos, convirtiéndose en una problema de salud pública por la alta prevalencia a nivel mundial $(1,6)$.

Según la OMS la prevalencia de caries dental se encuentra entre 60\%-90\% a nivel mundial, para el Perú la Oficina General de Epidemiología y Dirección General de Salud de las Personas en el año 2001 (último estudio epidemiológico realizado a nivel nacional por 
Tabla 2. Experiencia de caries dental según CPOD y CPOS en estudiantes de la Universidad Peruana Cayetano Heredia, Lima-Perú, en el año 2012.

\begin{tabular}{lccccc}
\hline \multicolumn{1}{c}{ FACULTADES } & POBLACIÓN & CPOD & & CPOS & \\
& N & X (DE) & p & X (DE) & p \\
\hline Ciencias y Filosofía & 436 & $4,5(3,3)$ & & $6,6(5,1)$ & \\
Enfermería & 371 & $4,2(3,6)$ & & $7,2(7,0)$ & \\
Estomatología & 474 & $5,0(3,4)$ & & $6,9(4,6)$ & \\
Medicina & 1135 & $4,9(3,6)$ & & $7,5(6,6)$ & \\
Medicina Veterinaria y Zootecnia & 272 & $4,1(3,0)$ & $<0,01^{*}$ & $6,4(5,5)$ & $0,01^{*}$ \\
Psicología & 163 & $4,8(3,2)$ & & $6,7(4,6)$ & \\
Salud Pública y Administración & 64 & $4,8(4,0)$ & & $11,1(11,7)$ & \\
Tecnología Médica & 501 & $4,9(3,7)$ & & $7,9(6,4)$ & \\
Total & 3417 & $4,7(3,5)$ & & $7,3(6,2)$ & \\
\hline
\end{tabular}

CPOD: Dientes permanentes cariados, perdidos y obturados.

CPOS: Superficies de dientes permanentes cariadas, perdidas y obturadas.

○: $\quad$ Escuela profesional dependiente de la Facultad de Medicina.

N: $\quad$ Población total.

X: Promedio.

DE: $\quad$ Desviación estándar.

p: $\quad$ Significancia estadística.

*: $\quad$ Prueba de Kruskall Wallis.

Base de Datos 2: 3417 registros de estudiantes.

el Ministerio de Salud del Perú) la prevalencia fue de $90,4 \%$ en edad escolar, los departamentos con mayor prevalencia fueron Ayacucho (99,8\%), Ica (98,8\%), Huancavelica $(98,3 \%)$ y Cusco $(97,2 \%)$, con respecto al área geográfica, la prevalencia en el área urbana fue $90,6 \%$ y en rural $88.7 \%(1,4,5)$.

Los resultados encontrados en el presente estudio se encuentran en el rango de prevalencia mundial dado por la OMS, sin embargo, se encuentra por debajo de la prevalencia a nivel nacional, para el Ministerio de Salud del Perú (MINSA) en Lima la prevalencia fue de $88,3 \%$ y Ancash, el departamento con menor prevalencia, reportó un $76,6 \%(4,5)$, es decir, en todos los casos los resultados de globales y por facultades de los estudiantes son más bajos que las prevalencias por departamentos. Las diferencias se deben a las características de los estudiantes, la universidad es una institución educativa privada y especializada en salud ubicada en Lima, la capital del país, los estudiantes cuentan con acceso a servicios de salud y constantes evaluaciones médicas y odontológicas preventivas.

La Organización Panamericana de Salud sugiere clasificar el Índice CPOD según su severidad en muy bajo $(0,0-1,1)$, bajo $(1,2-2,6)$, moderado $(2,7-4,4)$ y alto $(4,5-6,5)$ y muy alto $(6,6$ a más) (7).En la Universidad Peruana Cayetano Heredia se obtuvo un CPOD de 4,7 $\pm 3,5$, el cual se ubicó en un nivel alto de acuerdo a la clasificación establecida por la Organización Panamericana de Salud.

A nivel mundial, según el reporte de la OMS el Índice CPOD es aproximadamente 2,5 a los 12 años de edad, valor que se eleva en América Latina por encima de 3,5 siendo el continente con mayor promedio (1), según el MINSA en el año 2001, el Índice CPOD a los 12 años de edad fue de 3,67, siendo los departamentos con mayores índices CPOD fueron Ayacucho $(7,0)$, Ica $(5,3)$ y Amazonas $(5,0)$, y el de menor índice Áncash $(1,1)$, en Lima el valor del Índice CPOD fue de 2,2(3). En este caso el valor de los estudiantes de forma global o por facultades es siempre más alto al promedio nacional, y es más del doble del promedio de Lima, es importante mencionar que este indicador determina si el individuo ha sufrido de caries dental a lo largo de su vida, y estos estudiantes pueden haber tenido la enfermedad pero han debido tener 
una atención odontológica, lo cual demuestra la baja prevalencia pero la alta experiencia de caries dental.

En otros reportes, Álvarez A. y cols., en el año 1996, evaluaron 261 sujetos adultos en España presentando una prevalencia de caries de 99,6\% (8), por otro lado, en Colombia, López S. y col., en el 2011, realizaron un estudio con estudiantes de odontología, enfermería, bacteriología e instrumentación quirúrgica de la Corporación Universitaria Rafael Núñez del segundo periodo académico del 2010 en Cartagena, se mostró una prevalencia de caries dental de 56\% (9), similarmente, Arrieta K. y col., en el 2011, realizaron un estudio con 234 estudiantes del programa de Odontología de la Universidad de Cartagena, reportando una prevalencia de caries en los sujetos participantes de 55,9 \% (10). Al comparar los estudios descritos, las prevalencias de caries dental de los estudiantes de la Universidad Peruana Cayetano Heredia fueron más altos, una de las razones puede el tamaño poblacional de los estudios, el presente estudio cuenta con un total de 3454 registros de estudiantes, y de la heterogeneidad de la población, a pesar que todos son estudiantes de ciencias de la salud, la características de los estudiantes son diversas, las cuales cuáles podrían influenciar en los resultados.

También, Álvarez A. y col., reportaron un índice CPOD de 12,5, en ese estudio se pudieron obtener los promedio de los componentes: dientes cariados (DC $=2,9)$, dientes perdidos $(\mathrm{DP}=7,5)$ y sientes obturados $(\mathrm{DO}=2,1)$,este índice se considera en un nivel muy alto (8). Lamentablemente, para el presente estudio no se contó con los datos de los componentes del índice, como en el ejemplo, los dientes cariados indican la prevalencia de caries dental, los dientes perdidos y obturados el acceso a servicios de salud, una población con altos índices de CPOD, como la población estudiada, no necesariamente significa que las personas evaluadas tengan un alto daño de la enfermedad, eso sería sin los componentes de perdido y obturado fueran bajos en comparación con el de cares dental. $\mathrm{Y}$ al contrastar los indicadores de acceso a servicios de salud, un alto componente de diente perdido indica acceso a servicios poco preventivos o de poca capacidad resolutiva, al contrario de un alto indicador de diente obturado, que denota una respuesta oportuna al tratamiento odontológico.

El estudio realizado por Arévalo J. y col., en el año 2005 evaluaron a 271 estudiantes universitarios que asistieron a la Clínica Dental de la Dirección de Servicios Estudiantiles de Honduras, reportando un promedio de índice de CPOD de 14,6, siendo un nivel muy alto (11), al igual que el estudio de Cisneros M.y col., en el año 2009, donde 111 estudiantes universitarios del último año de licenciatura de la Facultad de Odontología en México alcanzaron un Índice CPOD de 9,73 (12). Estos valores difieren de los resultados de la presente investigación, inclusive al comparar a la Facultad de Estomatología que tiene el valor más alto de experiencia de caries dental.

Revisiones previas concuerdan con los resultados, si bien es cierto que los datos de prevalencia son altos en comparación con otras investigaciones, los valores del Índice CPOD y CPOS son bajos, esto es posible a la accesibilidad de los estudiantes a servicios de salud; por lo tanto, facilita y fortalece una cultura de prevención y promoción a la salud (13-15).

La investigación determinó la existencia de diferencias estadísticamente significativas de las prevalencias y experiencia de caries dental según facultades, un dato importante es indicar que la distribución de la prevalencia de caries dental fue acorde al costo de la educación por facultad (16), es decir, la más alta prevalencia de caries dental se evidenció en la Carrera Profesional de Tecnología Médica y en la Facultad de Enfermería, las cuales tienen las más bajas pensiones, al contrario de las Facultades de Estomatología, Ciencias y Filosofía, y Medicinaque registraron las prevalencias más bajas siendo los estudiantes con las más altas pensiones. Esto no ocurre de igual forma con el Índice CPOD, debido a que sólo indica número de dientes afectados por caries dental, pero si con el Índice CPOS que es un indicador de severidad y magnitud de la experiencia de caries dental, en este indicador la Facultad de Salud Púbica y Administración y la Carrera Profesional de Tecnología Médica tienen altos valores de superficies dentarias afectadas por caries dental, mientras que, las Facultades de Medicina Veterinaria y Zootecnia, Ciencias y Filosofía, y Estomatología tienen los promedios más bajos, igualmente en forma inversa al costo de la educación, es decir, al ser la UPCH una universidad privada y de las más costosas del Perú, este gasto en educación realizado por las familias de los estudiantes puede ser un indicador de diferencias socioeconómicas, esto confirma el efecto de la gradiente social sobre la salud de 
las personas (17), a pesar de estar supuestamente en igualdad de oportunidades.

Es conocido que, existen diferencias en los indicadores de salud según las clases sociales (18-21),Vásquez E. y col., señalan que hay una mayor prevalencia de caries dental en poblaciones con nivel socioeconómico bajo (22). Este factor económico puede tener un papel importante en el presente estudio, como lo menciona la Organización Colegial de Dentistas de España, mediante una encuesta de salud oral en el 2010, demostró que el nivel social y el lugar de residencia influyen en el índice de caries dental, sustentando que las personas de nivel socioeconómico alto tienen mayor oportunidad en el acceso a servicios de salud comparado a personas de nivel socioeconómico bajo (23-25).

Como se mencionó anteriormente, esta gradiente social se basa en los Determinantes Sociales de la Salud, siendo un nuevo paradigma en salud pública y considerada por la OMS como circunstancias en que las personas nacen, crecen, viven, trabajan y envejecen. Este paradigma reconoce que la gradiente social es la inequidad existente según la posición social en un grupo de personas repercutiendo en la salud de los mismos, y la cual siempre se repite a pesar de supuestas similitudes, esto es acorde con los resultados de la investigación (26-29).

Es importante determinar que el estudio contó con diversas limitaciones, las más resaltantes es que se ha basado en fuentes secundarias de datos, la heterogeneidad del tamaño de datos por facultades, la perdida de algunos datos por no tener los datos completos, sin embargo, la investigación permitió conocer la distribución de una enfermedad de alta prevalencia a nivel nacional y mundial, y además, la existencia de estas diferencias según facultades, si esto ocurre en universidad privada dedicada a ciencias de salud puede ser el mismo panorama en otras instituciones educativas privadas o estatales, por ello, debe ser de interés para poder las diversas autoridades establecer estrategias de solución a la problemática.

En conclusión, se evidenciaron diferencias estadísticamente significativas entre la prevalencia y la experiencia de caries dental en estudiantes según facultades de la Universidad Peruana Cayetano Heredia (UPCH), Lima-Perú, en el año 2012.

\section{Correspondencia:}

Miguel Espinoza Solano

Correo electrónico: miguel.espinoza@upch.pe

\section{REFERENCIA BIBLIOGRÁFICA}

1. World Health Organization. World Oral Health Report 2003. Ginebra: World Health Organization; 2004.

2. Ravindran S, Huang C, George A. Extracellular matrix of dental pulp stem cells: Applications in pulp tissue engineering using somatic MSCs. Frontiers in Psysiology. 2013; 6(4): 1-26.

3. Vera H, Valero M, Reyes A, Luengas E. Niñas y niños libres de caries en México. Revista ADM. 2010; 6754: 217- 22.

4. Ministerio de Salud. Prevalencia nacional de caries dental, fluorosis del esmalte y urgencia de tratamiento en escolares de 6 a 8, 10, 12 y 15 años. Lima, Perú: MINSA; 2001.

5. Ministerio de Salud. Documento Técnico Plan Nacional de Salud Bucal Sonríe Siempre Perú. 2001- 2002. Lima, Perú: MINSA; 2005.

6. Pérez-Luyo AG. ¿Es la caries dental una enfermedad infecciosa y transmisible?. Rev Estomatol Herediana. 2009; 19(2):118-124.

7. Nithila A, Bourgeois D, Barmes DE, Murtomaa H. Banco Mundial de Datos sobre Salud Bucodental de la OMS, 1986-1996: panorámica de las encuestas de salud bucodental a los 12 años de edad. Rev Panam Salud Publica. 1998; 4(6):411-5.

8. Alvarez-Arenal A, Alvarez-Riesgo JA, Pena JM, Fernandez JP, Villa MA. DMFT and treatment needs in adult population ot Oviedo, Spain. CommuntyDent Oral Epidemiol. 1996; 24: 17-20.

9. López J, Barrios K, Pallares L, Torres A, Torres D, Fortich N. Prevalencia de caries dental, factores de riesgo, enfermedad periodontal y hábitos de higiene oral en estudiantes de ciencias de la salud. Ciencia y Salud Virtual. 2011; 3(1): 35-41.

10. Arrieta K, Diaz A, Gonzalez F. Prevalencia de caries y enfermedad periodontal en estudiantes de odontología. Rev Cubana Estomatol.2001; 48(1): 6-13.

11. Arévalo S, Félix M, Rivera I, Sánchez F. Situación de la salud bucal de la población universitaria hondureña. Rev Med Hondur. 2005;73:161-5

12. Cisneros M, Tijerina L, Cantú P. La dieta y su importancia en la caries dental. Revista de Salud Pública y Nutrición. 2012; 13(3): 3-10.

13. De la Fuente-Hernández J,González M, Ortega-Maldonado M, Sifuentes-Valenzuela M. Caries y pérdida dental en estudiantes preuniversitarios mexicanos. Salud Publica Mex. 2008; 50: 235-40.

14. Piovano S, Squassi A, Bordoni N. Estado del arte de indicadores para la medición de caries dental. Rev 
UBA. 2010; 25(58): 29-41.

15. Henostroza G. Caries dental: Principios y procedimientos para el diagnóstico. Lima: Ripano Perú SAC; 2007.

16. Universidad Peruana Cayetano Heredia. Escala de pensiones por facultad (informes). Lima: Oficina de Admisión, Universidad Peruana Cayetano Heredia; 2015.

17. Wilkinson R, Marmot M. Los hechos probados. Ginebra: Organización Mundial de Salud; 2003.

18. Cavaillon J, Conge M, Mirisch D, Nemeth T, Sitbon J. Longitudinal study on oral health of dental students at Paris VII. University. Community Dent Oral Epidemiol, 1982: 10: 137-43.

19. Polk DE, Weyant RJ, Manz MC. Socioeconomic factors in adolescents' oral health: are they mediated by oral hygiene behaviors or preventive interventions? Community Dent Oral Epidemiol. 2010; 38: 1-9.

20. Pattussi MP, Hardy R, Sheiham A. The potential impact of neighborhood empowerment on dental caries among adolescents. Community Dent Oral Epidemiol 2006; 34: 344-50.

21. Aleksejuniene J, Eriksen HM, Holst D: Variation in caries and treatment experience in 35-44-year-old Lithuanians. Community Dent Oral Epidemiol. 2000; 28; 356-64.

22. Vázquez E, Calafell RA, Barrientos MC,et al.Prevalencia de caries dental en adolescentes: Asociación con género, escolaridad materna y estatus socioeconómico familiar. Rev.CES Odont.2011; 24(1): 17-22.

23. Consejo de Dentistas de España. Encuesta de Salud oral en España 2010. Madrid: Consejo de Dentistas de España; 2010. (Citado el 10 de enero 2014). Dispo- nible en: http://www.consejodentistas.es/pdf/DossierPrensa\%20EncuestaSaludOral2010.pdf .

24. Medina-Solís C, Maupomé G, Pelcastre-Villafuerte B, Avila-Burgos L,Vallejos-Sánchez A, CasanovaRosado A. Desigualdades socioeconómicas en salud bucal: Caries dental en niños de seis a 12 años de edad. Revista e Investigación Clínica. México. 2006; 58(4): $296-304$.

25. World Health Organization. Equity, social determinants and public health programmes. Ginebra: World Health Organization; 2010.

26. León-Manco R, Del Castillo-López C. Capital Social y experiencia de caries dental. Rev Estomatol Herediana.2011; 21(1):13-9.

27. Hadad-Arrascue N, Del Castillo-López C. Determinantes sociales de salud y caries dental. Odontol Pediatr. 2011; 10(1): 13-21.

28. Starfield B. Social gradients and child health. En: Heggenhuogen HK, Quah SR. International Encyclopedia of Public Health. San Diego CA: Academic Press; 2008.

29. Zárate G, Pérez M. Factores sociales como mediadores de la salud pública. Salu Uninorte. 2007; 23(2): 193-203.

Recibido : 27/12/2014

Aceptado: 10/06/2015 